\title{
Naked eye picometer resolution in a Michelson interferometer using conjugated twisted beams.
}

\author{
Olivier Emile ${ }^{1}$ and Janine Emile ${ }^{2}$ \\ ${ }^{1}$ Université de Rennes 1, 35042 Rennes Cedex, France.* \\ ${ }^{2}$ IPR, UMR 6251, CNRS, Université de Rennes 1, 35042 Rennes Cedex, France.
}

(Dated: January 11, 2017)

\begin{abstract}
Michelson interferometry is one of the most widely used techniques for accuracy measurements. Its main characteristic feature is to infer a displacement in one of the arm of the interferometer from a phase measurement. Two different twisted beams, also called vortex beams, with opposite twisted rotations in each arm of the interferometer interfere in a daisy flower like pattern. The number of petals is twice the topological charge. Their position depends on the relative phase of the beams. Naked eye detection of $44 \mathrm{pm}$ displacements is achieved. The sensitivity of such an interferometer together with possible further improvements and applications are then discussed.
\end{abstract}

OCIS Codes (080.4865) Optical vortices; (120.3180) Interferometry; (260.3160) Interference.

Denis Papin was one of the first to transform a linear displacement into a rotation [1], paving the way to the work of James Watt towards the development of condensing steam engines and more generally to modern heat engines [2]. On the other hand, electromagnetic fields possess characteristics associated with translational and rotational degrees of freedom $[3,4]$. The first ones are linked to the linear momentum that can be exchanged with matter via radiation pressure [5] and Doppler shifts [6], whereas the latter ones are linked to the angular momentum and can be exchanged via torques $[7,8]$ and rotational Doppler shifts $[9]$.

The detection of tiny linear displacements or translations is usually performed using interferometers such as Michelson interferometers [10-14]. They transform a length variation in one arm of the interferometer into a phase variation. Most of the time, the phase fronts are uniform and equal, leading to constructive or destructive interferences with dark or bright signals on the detector. Besides, high precision measurement requires complicated electronic treatments [15] and homodyne or heterodyne [16, 17] detections as well as accurate determination of the fringe location. One may then wonder what would be the response of the interferometer with non uniform phase fronts linked to the rotational degrees of freedom such as twisted beams [18] with different phase variations in the two arms. The purpose of this letter is to question the transfer of a linear mechanical displacement or a translation into a rotational degree of freedom of an electromagnetic field, and to investigate its detection in a Michelson interferometer.

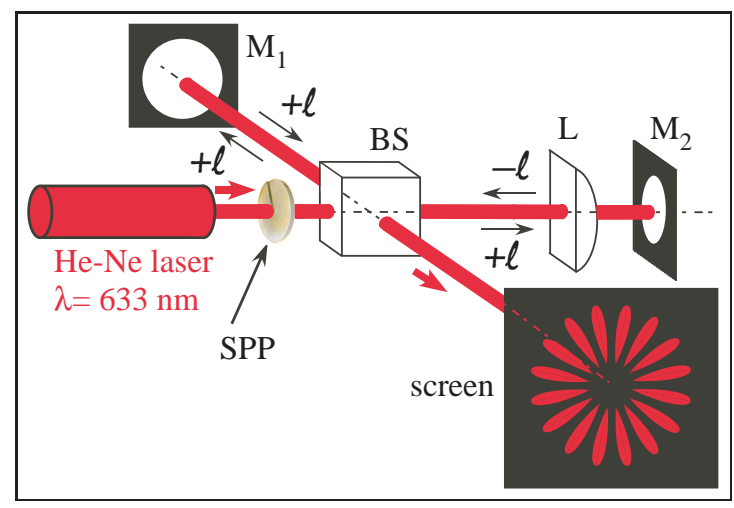

FIG. 1. Experimental set up. A Spiral Phase Plate (SPP) transforms the fundamental mode of a He-Ne laser into a LG beam before entering the Michelson interferometer. M1 and M2: mirrors mounted on microstages and piezo-electric translations. BS: Beam Splitter. A cylindrical lens (L) reverses the topological charge $+\ell$ of the beam to $-\ell$, in one of the arms. The interference between $+\ell$ and $-\ell$ at the output of the interferometer is a daisy flower, seen on a screen. The picture corresponds here to $\ell=8$.

A slightly modified Michelson interferometer (see Fig. 1) is operated to probe tiny dis- 
placements of one mirror. The whole set-up lies on an optical table. Microstages and piezoelectric transducers control the length of the 30-cm-long arms of the interferometer. The source is a He-Ne laser (Melles-Griot, $\mathrm{P}=1$ $\mathrm{mW}, \lambda=633 \mathrm{~nm})$. The interferometer is first adjusted on a dark fringe. Then, the fundamental mode of the laser is transformed into a higher order Laguerre Gaussian (LG) beam with a Spiral Phase Plate (SPP, RC Photonics) [19]. Such beams have a phase that is not uniform in a plane perpendicular to the direction of propagation. It varies as $\ell \theta[18], \theta$ being the azimuthal angle and $\ell$ the so-called topological charge. Besides, in comparison with fundamental Gaussian beams, their spatial extension is larger and the heating of the mirrors of the interferometer is known to be reduced [20]. Finally, a cylindrical astigmatic lens ( $\mathrm{f}=$ $10 \mathrm{~cm}$ ) is added in one arm of the interferometer. The distance between the lens and the mirror equals the focal length of the lens. It reverses the topological charge of the beam from $+\ell$ to $-\ell[21]$. Then the $+\ell$ and $-\ell$ conjugate beams are combined after the beam splitter and interfere on a screen.

The amplitude of the electric field of a LG beam at a distance $z$ from the beam waist $w_{0}$ and for a radial distance $r$ can be written as [18]

$E_{\ell}(r, \theta, z)=\left[\begin{array}{l}\frac{C}{w(z)}\left[\frac{r}{w(z)}\right]^{|\ell|} \exp \left[\frac{-r^{2}}{w^{2}(z)}\right] \times \\ L^{|\ell|}\left[\frac{2 r^{2}}{w^{2}(z)}\right] \exp (i \varphi(r, z)) \exp (i \ell \theta)\end{array}\right]$

$C$ being a normalization constant, $L^{|\ell|}$ the $\ell$ Laguerre polynomial, $\varphi(r, z)$ a phase term related to the propagation and

$$
w(z)=w_{0} \sqrt{1+z^{2} / z_{R}^{2}}
$$

refers to the typical beam radius, with $z_{R}=$ $\pi w_{0}^{2} / \lambda$ being the so-called Rayleigh range, and $\lambda$ the wavelength. The amplitude can be written in a simpler form

$$
E_{\ell}(r, \theta)=R_{\ell}(r) \exp (i \ell \theta)
$$

$R_{\ell}(r)$ includes the $z$ and the radial dependence of the field of Eq. 1. It also depends on the absolute value of $\ell$. The expression of the amplitude of a $-\ell$ LG beam only includes a minus sign in the " $\theta$ " dependence of Eq. 3 compared with a $\ell$ LG beam. The expression of the total field, combination of the two $+\ell$ and $-\ell$ conjugate LG beams of the two arms of the interferometer, assuming the same path in the two arms, is then

$E_{\ell}(r, \theta)+E_{-\ell}(r, \theta)=2 R_{\ell}(r) \exp (i \ell \theta / 2) \cos \ell \theta$

The light intensity $I(r)$ then writes

$$
I(r)=4\left|R_{\ell}(r)\right|^{2} \cos ^{2} \ell \theta
$$

There is thus an angular modulation of the intensity of the resulting field. The exact expression of the electric field and the resulting intensity distribution has been calculated recently [22], taking into account the radial dependence of the field. The authors found a $1+\cos \ell 2 \theta$ modulation of the intensity which is strictly speaking equivalent to the $\cos ^{2} \ell \theta$ here. They plotted the intensity distribution and found a daisy flower interference pattern like the one found here. This is similar to what is observed in entanglement experiments [23, 24]. This is evidenced on Fig. 2 where the origin of the azimuthal angle $\theta$ could be chosen arbitrarily. The number of petals is twice the topological charge of the beam $(2 \ell)$. Figures $2 \mathrm{a}, \mathrm{c}$, e, correspond to the experimental pictures of the daisy flowers for $\ell=1,2,8$ respectively. Figures $2 \mathrm{~b}$, d, f are the corresponding simulated intensities according to equation 5 .

Let us introduce an extra distance $\delta$ in the length of one of the arms of the interferometer, in the arm with the $\ell$ beam for example. The expression of the resulting electric field becomes

$$
\begin{aligned}
& E_{\ell}(r, \theta)+E_{-\ell}(r, \theta)=R_{\ell}(r)[\exp i(\ell \theta+2 k \delta)+\exp (-i \ell \theta)] \\
& =2 R_{\ell}(r) \exp (i \ell \theta / 2) \exp (i k \delta) \cos (\ell \theta+k \delta)
\end{aligned}
$$

where $k=2 \pi / \lambda$ is the wave vector modulus. Then, the daisy flower is rotated by an angle $2 \pi \delta / \lambda$. This ratio between the pattern rotation angle and the mirror displacement $2 \pi / \lambda$ is very large. this enables tiny displacement measurements from the pattern rotation angle. Whereas in a usual Michelson interferometer, a variation of the path in one of the arm 


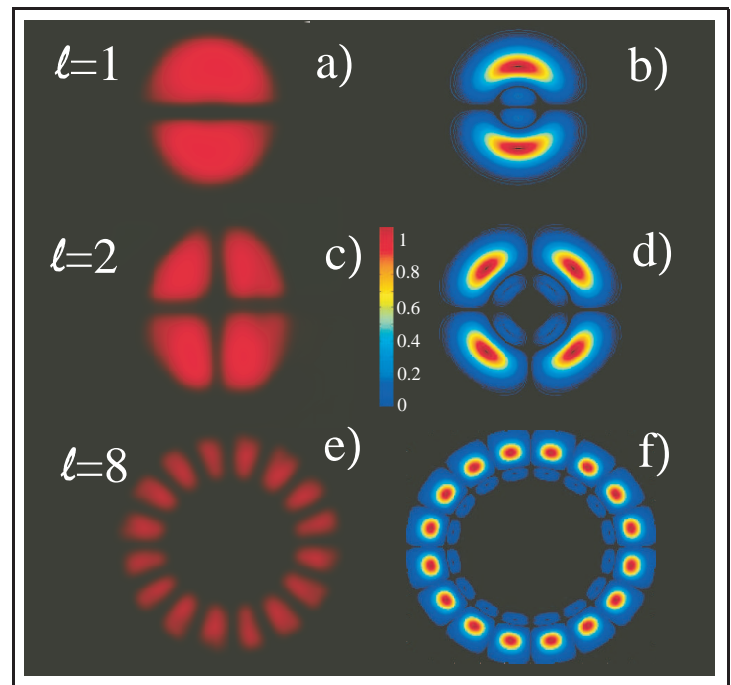

FIG. 2. Experimental daisy flowers of the interference pattern (a, c, e) and corresponding normalized simulated intensities $(\mathrm{b}, \mathrm{d}, \mathrm{f})$. The $+\ell$ and $-\ell$ conjugate beam superposition $(\ell=1,2,8)$ leads to interference patterns which number of petals is twice the topological charge $\ell$. Note that the zero azimuthal angle could be chosen arbitrarily. It depends on the initial relative phase between the two arms. Here the origin is not the same for $\ell=1$, $\ell=2$ and $\ell=8$.

leads to a modulation of the detected intensity that can be subjected to intensity fluctuations of the source, contrarily here, the same variation of the path leads to a rotation of the interference pattern. The Michelson interferometer is operated in a very different way. The total light intensity on the screen is constant. Only its spatial intensity distribution may be rotated.

In order to check this assumption, the optical path in one arm with the piezo-electric stage has been slightly changed. The pattern rotation angle versus the mirror displacement is displayed on Fig. 3. One notes that there is a perfect linear dependence of the rotation angle versus the displacement. From a least mean square method, the coefficient equals $568.37 \pm 1.14^{\circ} / \mu \mathrm{m}(9.92 \pm 0.02 \mathrm{rad} / \mu \mathrm{m})$, in excellent agreement with the expected value $2 \pi / \lambda=9,9283 \mathrm{rad} / \mu \mathrm{m}$ of Eq. 6 .

Besides, looking at the dynamics of the ro-

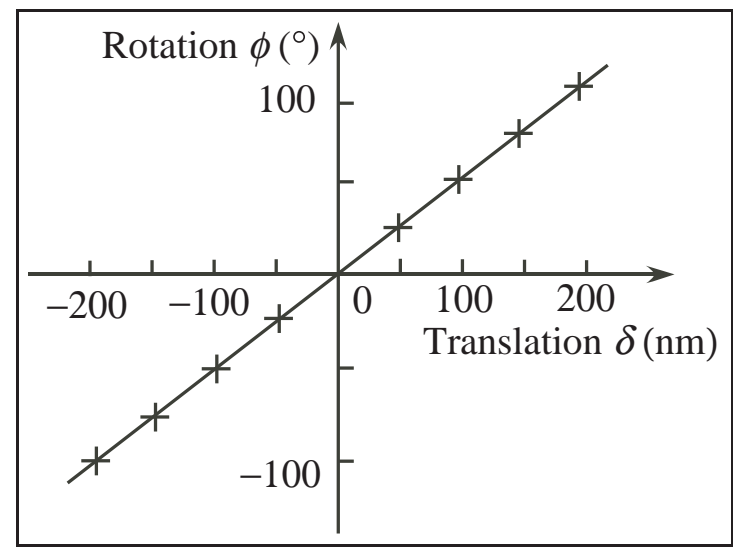

FIG. 3. Experimental rotation of the 16-petals flower $(\ell=8)$ versus mirror displacement. Crosses: experimental values, straight line: numerical fit of the data, showing a perfect linear variation.

tation, there is the same linear dependence between the displacement velocity of mirror $\mathrm{M}_{1}$ and the angular velocity rotation of the flower, as expected. This can also be understood in reasoning in the frequency domain. The two conjugate beams interfer at the output of the Michelson interferometer. Since one mirror moves at a given velocity, the frequency of one of the $\ell$ beams is Doppler shifted. The two beams have not the same frequency any more. Thus the interference between the two beams is rotating at a frequency that equals the frequency shift. The linear velocity of the mirror is transposed into a rotational velocity that is evidenced on the daisy flower.

One may then wonder what could be the resolution of such an interferometer. Michelson interferometer is known to be one of the most sensitive interferometers to detect tiny displacements $[12,13,15]$. Usually, to gain much sensitivity, it operates close, but not exactly at total extinction. Then, the optical power within the interferometer has to be maximal to minimize the noise, leading to sophisticated electronics, recycling cavities and radiation pressure problems. More precisely, power fluctuations induce fluctuations on the exact position close to a dark fringe. Moreover, more generally, signal to noise ration for 
optical sensing varies as the square root of the optical power [12]. This power increase means additional radiation pressure noise and thermal load inside the cavity [20]. To circumvent this problem, conventional sources may be replaced by more complicated sources such as squeezed light [13].

Here, the output signal is fundamentally different. There is no bright or dark fringes. There is a daisy flower which petal position only depends on the relative phase between the two conjugate LG beams. The displacement of one of the mirror is transposed into the rotation of the daisy flower. Then, the sensitivity is directly linked to the quality of the detection of the rotation of the daisy flower. For a $\ell=8$ topological charge, with a daisy flower of $d=2$ $\mathrm{m}$ diameter, displacements of $\delta=0.88 \mathrm{~nm}$ are detected, as can be seen in Fig. 4a. Operating in a darkness environment, A naked eye detection reaches, a $\delta=44 \mathrm{pm}$ displacement measurement (see Fig. 4b). The detection is performed here on the rotation of a single petal of the daisy flower. Actually, it is achieved on the variation of the position of the intensity distribution. Therefore steep intensity distribution should be used with high $\ell$ values. The higher the $\ell$, the thinner the petal, the easier the detection.

What could be the ultimate sensitivity of such a detection scheme? One can expend our image, increase the laser power in order to gain higher contrast and also increase the topological charge of the beam. LG beams up to $\ell=300$ have been realized recently [23] leading to a daisy with potentially 600 petals. Each petal must then be $\pi d / 600=1 \mathrm{~mm}$ wide. A Position Sensitive Detector (PSD) could measure optical beam shifts up to $1 \mathrm{~nm}$ [25]. It would then correspond to a rotation angle of $\phi=10^{-9} \mathrm{rad}$, and a displacement of the mirror of $10^{-16} \mathrm{~m}$ here.

Indeed, the uncertainty of a detector such as a PSD is a length divided by the square root of the number $n$ of the detected photons [26]. Assuming a detection over the whole circumference of the daisy flower, this would imply an uncertainty of the phase of $2 \pi / \sqrt{n}$. This is the usual phase uncertainty considered for

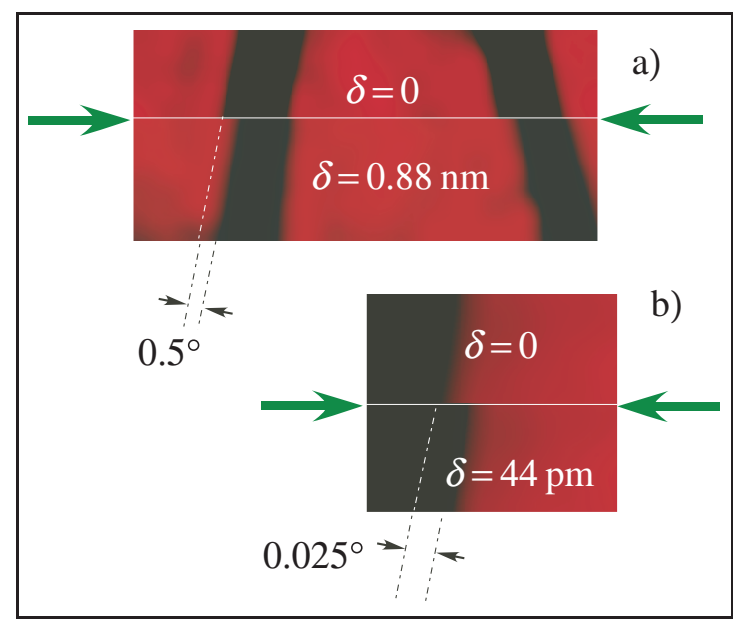

FIG. 4. Sensitivity of the detection. Zoom pictures of the daisy flowers $(\ell=8)$ with zero displacement (top) and $\delta$ displacement (bottom) have been superposed to highlight the sensitivity. a) $0.88 \mathrm{~nm}$ displacement. b) $44 \mathrm{pm}$ displacement.

Michelson interferometers. Nevertheless, here, the detection is not performed close to the extinction fringe. This means that the number of detected photons could be much higher, leading to a lower uncertainty. It has however to be noted that some attempts have been made to make a detection closer to the maximum intensity fringes in the Michelson interferometer [27].

What could be a reasonable experimental uncertainty in our case? With a $2 \mathrm{~W}$ laser, let us assume that $1 \mathrm{~W}$ is detected. The rest of the injected power exits the interferometer on the other face of the beam splitter. At $\lambda=633 \mathrm{~nm}$ and at a measurement rate of $1 \mathrm{~Hz}$, the number of useful photons $n$ is equal to the power $P$ divided by the measurement rate divided by the energy of a single photon $(n=P \times \lambda / h c, c$ being the light velocity, and $h$ being the Planck constant). It is of the order of $3 \times 10^{18}$. This leads to an uncertainty on the phase of the order of $3 \times 10^{-9} \mathrm{rad}$. In order to increase this uncertainty, one would have to increase the number of photon. This means to either increase the power or the wavelength.

Nevertheless, one has to be very cautious when increasing the beam topological charge 
as suggested above, since the beam would then strongly diverges [28]. However, according to Eqs. 1 and 2, if one stays below the Rayleigh distance, the beam size will remain nearly constant. For example, for a cavity length $d=z_{R}=0.5 \mathrm{~m}$ and a wavelength $\lambda=633$ $\mathrm{nm}$, one finds a waist of the order of $w_{0} \approx 0.3$ $\mathrm{mm}$. The typical size of a LG beam varies as $r_{\max }=w_{0} \sqrt{\ell / 2}[28]$. For $\ell=300$, this leads to $r_{\max }=12 w_{0}$ which is reasonable, whereas for $\ell=10000$ [24] this leads to $r_{\max }=100 w_{0}$. Larger mirrors (of the order of $1 \mathrm{~m}$ ) have then to be used. For $1 \mathrm{~km}$ long arms the use of $\ell=10000$ beams would be meaning less. The mirrors would be too large.

In conclusion, a modified 30-cm-long Michelson interferometer using conjugate twisted beams leads to a "naked eye" picometer resolution. The interference pattern is a daisy flower like picture that rotates under tiny translations. The number of petals is twice the topological charge of the beam. The mirror translation is transposed into a rotational degree of freedom of the beam. The ratio between the rotation angle $2 \pi / \lambda$ is very large, paving the way to tiny displacement measurements from pattern rotations. 44 pm resolutions have been obtained. With further improvements, using commercially available PSD, the atto meter detection range seems within reach.

These detection techniques using the interference pattern of two opposite charge twisted beam can also be operated in nearly every Michelson interferometer and may thus be used for further developments. For example, in a modern version of the Michelson Morley experiment, it would enable to test fundamental relativistic effects $[29,30]$. Besides, since the interferometer is strong and compact, it can be directly implemented in modern gravimeters used in geophysics [31, 32], in optical coherence tomography systems used in biology [33, 34], or in helioseismology studies [35].

\section{ACKNOWLEDGMENTS}

Acknowledgment. The authors thank Jean René Thébault for technical assistance.

* Corresponding author; olivier.emile@univrennes1.fr

[1] A.A. Ernouf, Denis Papin sa vie et son oeuvre 1647-1714, (Hachette, Paris, 1874).

[2] D.P. Miller. James Watt, chemist: Understanding the origins of the steam age, (Routledge, New York, 2015).

[3] B. Thidé, F. Tamburini, H. Then, C.G. Someda, and R.A. Ravanelli, The physics of angular momentum radio. arXiv preprint arXiv:1410.4268 (2014).

[4] A. Aiello, P. Banzer, M. Neugebauer, and G. Leuchs, "From transverse angular momentum to photonic wheels" Nat. Photon. 9, 789-795 (2015).

[5] A. Ashkin, "Applications of laser radiation pressure" Science 210, 1081 (1980).

[6] A. Eden, The Search for Christian Doppler (Springer, Vienna, 1992).

[7] R.A. Beth, "Direct detection of the angular momentum of light" Phys. Rev. 48, 471 (1935).

[8] O. Emile, C. Brousseau, J. Emile, R. Niemiec, K. Madhjoubi, and B. Thidé, "Electromagnetically induced torque on a large ring in the microwave range" Phys. Rev. Lett. 112, 053902 (2014).

[9] J. Courtial, D.A. Robertson, K. Dholakia, L. Allen, and M.J. Padgett, "Rotational frequency shift of a light beam" Phys. Rev. Lett. 81, 4828 (1998).

[10] A. Michelson and E. Morley, "on the relative motion of the earth and the luminiferous ether" Am. J. Sci. 34, 333 (1887).

[11] J. Lawall and E. Kessler, "Michelson interferometry with 10 pm accuracy" Rev. Sci. Instrum. 71, 2669 (2000).

[12] P. Hariharan, Optical Interferometry. 2nd ed. (Academic Press, San Diego, 2003).

[13] R.X. Adhikari, "Gravitational radiation detection with laser interferometry" Rev. Mod. Phys. 86, 121 (2014).

[14] K.G. Libbrecht and E.D.A Black, "A basic Michelson laser interferometer for the undergraduate teaching laboratory demonstrating picometer sensitivity" Am J. Phys. 83, 409 (2015). 
[15] LIGO Scientific Collaboration and VIRGO Collaboration, "GW150914: The Advanced LIGO Detectors in the Era of First Discoveries" Phys. Rev. Lett. 116, 131103 (2016).

[16] M.J. Connelly, J.H. Galeti, and C. Kitano, Michelson interferometer vibrometer using self-correcting synthetic-heterodyne demodulation" Appl. Opt. 54, 5734 (2015).

[17] Z. Cheng, D. Liu, Y. Zhou, Y. Yang, J. Luo, Y. Zhang, Y. Shen, C. Liu, J. Bai, K. Wang, L. Su, and L. Yang, "Frequency locking of a field-widened Michelson interferometer based on optimal multi-harmonics heterodyning." Opt. Lett. 41, 3916 (2016).

[18] G. Molina-Terriza, J.P. Torres, and L. Torner, "Twisted photons" Nat. Phys. 3, 305 (2007).

[19] M.W. Beijersbergen, R.C.P. Coerwinkel, M. Kristensen, and J.P. Woerdman, "Helicalwavefront laser beams produced with a spiral phaseplate" Opt. Comm. 112, 321 (1994).

[20] L. Carbone, C. Bogan, P. Fulda, A. Freise, and B. Willke, "Generation of high-purity higher-order Laguerre-Gauss beams at high laser power" Phys. Rev. Lett. 110, 251101 (2013).

[21] M.J. Padgett and L. Allen, "Orbital angular momentum exchange in cylindrical-lens mode converters" J. Opt. B: Quantum Semicl. Opt. 4, S17 (2002).

[22] J. Guo, B. Guo, R. Fan, W. Zhang, Y. Wang, L. Zhang, and P. Zhang, "Measuring topological charges of Laguerre-Gaussian vortex beams using two improved Mach-Zehnder interferometers." Opt. Eng. 55, 035104 (2016).

[23] R. Fickler, R. Lapkiewicz, W.N. Plick, M. Krenn, C. Schaeff, S. Ramelow, and A. Zeilinger, "Quantum entanglement of high angular momenta" Science 338 , 640 (2012).

[24] R. Fickler, G. T. Campbell, B. C. Buchler, P. K. Lam, and A. Zeilinger, Quantum entanglement of angular momentum states with quantum numbers up to 10010, arXiv preprint ArXiv: 1607.00922 (2016).

[25] J. Emile, A. Sane, H. Tabuteau, and O. Emile, Soft Matter "Experimental investiga- tion of a moving contact line in a channel" $\mathbf{9}$, 10229 (2013).

[26] S. Donati, C.-Y. Chen, and C.-C. Yang, "Uncertainty of positioning and displacement measurements in quantum and thermal regimes" IEEE Trans. Instrum. Meas. 56, 1658 (2007).

[27] D. Grassani, M. Galli, and D. Bajoni, "Active stabilization of a Michelson interferometer at an arbitrary phase with subnanometer resolution" Opt. Lett. 39, 2530 (2014).

[28] M. J. Padgett, F. M. Miatto, M. P. J. Lavery, A. Zeilinger, and R. W. Boyd, "Divergence of an orbital-angular-momentum-carrying beam upon propagation" New J. Phys. 17, 023011 (2015).

[29] Ch. Eisele, A.Y Newsky, and S. Schiller, "Laboratory test of the isotropy of light propagation at the $10^{-17}$ level" Phys. Rev. Lett. 103, 090401 (2009).

[30] M. Nagel, S.R. Parker, E.V. Kovalchuk, P.L. Stanwix, J.G. Hartnett, E.N. Ivanov, A. Peters, and M.E. Tobar, "Direct terrestrial test of Lorentz symmetry in electrodynamics to $10^{-18 "}$ Nat. Commun. 6, 8174 (2015).

[31] J.P. Schwarz, D.S. Robertson, T.M. Niebauer, and J.E. Faller, "A free-fall determination of the Newtonian constant of gravity" Science 282, 2230 (1998).

[32] H. Baumann, F. Pythoud, D. Blas, S. Sibiryakov, A. Eichenberger, and E.E. Klingelé, "Experimental assessment of the speed of light perturbation in free-fall absolute gravimeters" Metrologia 52, 635 (2015).

[33] D. Huang, E.A. Swanson, C.L. Lin, J.S. Schuman, W.G. Stinson, W. Chang, M.R. Hee, T. Flotte, K. Gregory, C.A. Puliafito, and J.G. Fujimoto, "Optical coherence tomography" Science 254, 1178 (1991).

[34] A. M. Rollins, and J. A. Izatt, "Optimal interferometer designs for optical coherence tomography" Opt. lett. 24, 1484 (1999).

[35] S. Ilonidis, J. Zhao, and A. Kosovichev, "Detection of emerging sunspot regions in the solar interior" Science 333, 993 (2011). 